\title{
Non-Symmetric Basic Hypergeometric Polynomials and Representation Theory for Confluent Cherednik Algebras
}

Marta MAZZOCCO

Department of Mathematical Sciences, Loughborough University, Loughborough LE11 3TU, UK E-mail: m.mazzocco@lboro.ac.uk

URL: http://homepages.Iboro.ac.uk/ mamm4/

Received October 31, 2014, in final form December 19, 2014; Published online December 30, 2014 http://dx.doi.org/10.3842/SIGMA.2014.116

Abstract. In this paper we introduce a basic representation for the confluent Cherednik algebras $\mathcal{H}_{\mathrm{V}}, \mathcal{H}_{\mathrm{III}}, \mathcal{H}_{\mathrm{III}}^{D_{7}}$ and $\mathcal{H}_{\mathrm{III}}^{D_{8}}$ defined in arXiv:1307.6140. To prove faithfulness of this basic representation, we introduce the non-symmetric versions of the continuous dual $q$ Hahn, Al-Salam-Chihara, continuous big $q$-Hermite and continuous $q$-Hermite polynomials.

Key words: DAHA; Cherednik algebra; $q$-Askey scheme; Askey-Wilson polynomials

2010 Mathematics Subject Classification: 33D80; 33D52; 16T99

\section{Introduction}

In this paper we introduce a faithful representation on the space $\mathcal{A}$ of Laurent polynomials for the confluent Cherednik algebras $\mathcal{H}_{\mathrm{V}}, \mathcal{H}_{\mathrm{III}}, \mathcal{H}_{\mathrm{III}}^{D_{7}}$ and $\mathcal{H}_{\mathrm{III}}^{D_{8}}$ defined in $[5]^{1}$ as confluences of the Cherednik algebra of type $\check{C}_{1} C_{1}[2,6,7]$ :

- $\mathcal{H}_{\mathrm{V}}$ is the algebra generated by $T_{0}, T_{1}, X^{ \pm 1}$ with relations:

$$
\begin{aligned}
& \left(T_{1}+a b\right)\left(T_{1}+1\right)=0, \\
& T_{0}\left(T_{0}+1\right)=0 \\
& \left(T_{1} X+a\right)\left(T_{1} X+b\right)=0, \\
& q T_{0} X^{-1}+c=X\left(T_{0}+1\right) .
\end{aligned}
$$

- $\mathcal{H}_{\mathrm{III}}$ is the algebra generated by $T_{0}, T_{1}, X^{ \pm 1}$ with relations:

$$
\begin{aligned}
& \left(T_{1}+a b\right)\left(T_{1}+1\right)=0, \\
& T_{0}^{2}=0 \\
& \left(T_{1} X+a\right)\left(T_{1} X+b\right)=0 \\
& q T_{0} X^{-1}+1=X T_{0} .
\end{aligned}
$$

- $\mathcal{H}_{\mathrm{III}}^{D_{7}}$ is the algebra generated by $T_{0}, T_{1}, X^{ \pm 1}$ with relations:

$$
\begin{aligned}
& T_{1}\left(T_{1}+1\right)=0, \\
& T_{0}^{2}=0,
\end{aligned}
$$

\footnotetext{
${ }^{1}$ See [5, Theorem 4.1] for $\mathcal{H}_{\mathrm{V}}, \mathcal{H}_{\mathrm{III}}$ and [5, Definition 1.4] for $\mathcal{H}_{\mathrm{III}}^{D_{7}}$ and $\mathcal{H}_{\mathrm{III}}^{D_{8}}$ - observe that in [5] $W$ is $X^{-1}$ for these algebras.
} 


$$
\begin{aligned}
& T_{1} X+a-X^{-1}\left(T_{1}+1\right)=0 \\
& q T_{0} X^{-1}+1-X T_{0}=0
\end{aligned}
$$

- $\mathcal{H}_{\mathrm{III}}^{D_{8}}$ is the algebra generated by $T_{0}, T_{1}, X^{ \pm 1}$ with relations:

$$
\begin{aligned}
& T_{1}\left(T_{1}+1\right)=0, \\
& T_{0}^{2}=0, \\
& T_{1} X-X^{-1}\left(T_{1}+1\right)=0, \\
& q T_{0} X^{-1}+1-X T_{0}=0 .
\end{aligned}
$$

To prove faithfulness of our basic representation (see Theorems 2.2, 3.2 and 4.2 here below) in each case, we select a special basis of polynomials in $\mathcal{A}$ on which the operators (or specific combinations of them) act nicely. These bases are obtained by considering the non-symmetric versions of the continuous dual $q$-Hahn, Al-Salam-Chihara, continuous big $q$-Hermite and continuous $q$-Hermite polynomials respectively.

In [7] Sahi introduced the non-symmetric version of Koornwinder polynomials [1], and proved that they form a basis in the space $\mathcal{A}$ of Laurent polynomials. A detailed discussion of the rank one case, i.e. the non-symmetric Askey-Wilson polynomials, was presented in [6] (see also [4]). It turns out that these non-symmetric Askey-Wilson polynomials behave well under the subsequent degeneration limits $d \rightarrow 0, c \rightarrow 0, b \rightarrow 0$ and finally $a \rightarrow 0$. However the proof of faithfulness of our basic representation is not a straightforward limit of the same proof in the case of the Askey-Wilson algebra, as one would naively expect. This is because the first degeneration limit destroys some of the leading coefficients in the positive powers of $z$ of half the non-symmetric continuous dual $q$-Hahn polynomials and their degenerations. Moreover, the algebra $\mathcal{H}_{\text {III }}$ is not in fact the limit of $\mathcal{H}_{\mathrm{V}}$ as $c \rightarrow 0$ but the one as $c \rightarrow \infty$, which introduces the need of an

isomorphism and a few tricks. Last but not least, the $\mathcal{H}_{\mathrm{III}}^{D_{7}}$ and $\mathcal{H}_{\mathrm{III}}^{D_{8}}$ do not admit a presentation à la Bernstein-Zelevinsky, which makes the proof of faithfulness in that case rather involved.

\section{Non-symmetric continuous dual $q$-Hahn polynomials and basic representation of $\mathcal{H}_{\mathrm{V}}$}

The continuous dual $q$-Hahn polynomials are the following (we write them here in monic form like in $[3])$ :

$$
p_{n}(z ; a, b, c):=\frac{(a b, a c ; q)_{n}}{a^{n}}{ }_{3} \phi_{2}\left(\begin{array}{c}
q^{-n}, a z, a z^{-1} \\
a b, a c
\end{array} ; q, q\right),
$$

and can be obtained from the Askey-Wilson polynomials as limits when $d \rightarrow 0$. This same limit can be performed on the non-symmetric the Askey-Wilson polynomials, leading to the following (here we follow [4] approach):

Definition 2.1. Let

$$
q_{n}^{\dagger}(z ; a, b, c):=q^{\frac{n-1}{2}}(z-c) p_{n-1}\left(q^{-\frac{1}{2}} z ; q^{\frac{1}{2}} a, q^{\frac{1}{2}} b, q^{\frac{1}{2}} c\right),
$$

the non-symmetric continuous dual $q$-Hahn polynomials are defined as follows:

$$
\begin{aligned}
& E_{-n}[z]:=p_{n}(z ; a, b, c)-q_{n}^{\dagger}(z ; a, b, c), \quad n=1,2, \ldots, \\
& E_{n}[z]:=q^{n} p_{n}(z ; a, b, c)+\left(1-q^{n}\right) q_{n}^{\dagger}(z ; a, b, c), \quad n=1,2, \ldots, \\
& E_{0}[z]:=1 .
\end{aligned}
$$


Theorem 2.2. For $q, a, b, c \neq 0, q^{m} \neq 1(m=1,2, \ldots)$, the algebra $\mathcal{H}_{\mathrm{V}}$ has a faithful representation on the space $\mathcal{A}$ of Laurent polynomials $f[z]$ as follows:

$$
\begin{aligned}
& \left(T_{0} f\right)[z]:=\frac{(z-c) z}{q-z^{2}}\left(f[z]-f\left[q z^{-1}\right]\right), \\
& \left(T_{1} f\right)[z]:=\frac{(a+b) z-(1+a b)}{1-z^{2}} f[z]+\frac{(1-a z)(1-b z)}{1-z^{2}} f\left[z^{-1}\right], \\
& (X f)[z]:=z f[z] .
\end{aligned}
$$

To prove this theorem we follow the same outline as the proof of Theorem 5.3 in [4] with some important changes as explained in Remark 2.5 here below.

First of all, to prove that the operators defined by (2.1)-(2.3) satisfy the relations (1.1)-(1.4) is a straightforward computation. To prove faithfulness, we need the following two lemmata:

Lemma 2.3. Let

$$
Z:=\left(T_{0}+1\right) T_{1}^{-1} \quad \text { and } \quad Y:=T_{1} T_{0},
$$

the algebra $\mathcal{H}_{\mathrm{V}}$ can equivalently be described as the algebra generated by $T_{1}, X^{ \pm 1}, Y, Z$, satisfying the following relations respectively:

$$
\begin{aligned}
& Z Y=Y Z=0, \\
& X T_{1}=-a b T_{1}^{-1} X^{-1}-a-b, \\
& T_{1}^{-1} Y=Z T_{1}-1, \\
& \left(T_{1}+a b\right)\left(T_{1}+1\right)=0, \\
& a b Y X=-q T_{1}^{2} X Y-q(a+b) T_{1} Y-a b T_{1} X+a b c T_{1} .
\end{aligned}
$$

The algebra $\mathcal{H}_{V}$ is spanned by elements $X^{m} Y^{n} T_{1}^{i}$ and $X^{m} Z^{n} T_{1}^{i}$, where $m \in \mathbb{Z}, n \in \mathbb{N}$ and $i=1,2$.

Proof. To prove the equivalence it is enough to observe that by defining $Z$ and $Y$ as in (2.4), relations (2.5)-(2.9) follow from (1.1)-(1.4). Vice-versa, defining $T_{0}:=T_{1}^{-1} Y$ we see that relations $(2.5)-(2.9)$ imply (1.1)-(1.4).

To prove that $\mathcal{H}_{V}$ is spanned by elements $X^{m} Y^{n} T_{1}^{i}$ and $X^{m} Z^{n} T_{1}^{i}$, where $m \in \mathbb{Z}, n \in \mathbb{N}$ and $i=1,2$, we use the relations (2.5)-(2.9) and the further relations which can be obtained as a consequence of $(2.5)-(2.8)$ :

$$
\begin{aligned}
Y X^{-1} & =q^{-1} X^{-1} Y+q^{-1}(1+a b) X^{-1} Z T_{1}-q^{-1}(a+b) Z T_{1}+q^{-1} X^{-1} T_{1}-c q^{-1} T_{1}, \\
Z X= & q^{-1} X Z-q \frac{1+a b}{a b} X^{-1} Z T_{1}+\frac{a+b}{a b} Z T_{1}-\frac{1}{a b} X^{-1} T_{1} \\
& +\frac{c}{a b q} T_{1}+\frac{(1+a b)(q-1)}{a b}\left(X^{-1}-\frac{c}{q}\right)
\end{aligned}
$$

to order any word in the algebra as wanted.

Lemma 2.4. The non-symmetric continuous dual q-Hahn polynomials form a basis in the space $\mathcal{A}$ of Laurent polynomials and are eigenfunctions of the operators $Y:=T_{1} T_{0}$ and $Z:=\left(T_{0}+\right.$ 1) $T_{1}^{-1}$ :

$$
\begin{aligned}
& \left(Y E_{-n}\right)[z]=\frac{1}{q^{n}} E_{-n}[z], \quad n=1,2,3, \ldots, \\
& \left(Y E_{n}\right)[z]=0, \quad n=0,1,2, \ldots
\end{aligned}
$$




$$
\begin{aligned}
& \left(Z E_{-n}\right)[z]=0, \quad n=1,2,3, \ldots, \\
& \left(Z E_{n}\right)[z]=-\frac{1}{a b q^{n}} E_{n}[z], \quad n=0,1,2, \ldots
\end{aligned}
$$

Proof. By using the definition of the $q$-hypergeometric series ${ }_{3} \phi_{2}$ it is easy to prove that the terms with the highest powers in $z$ and $\frac{1}{z}$ in $E_{-n}$ and $E_{n}$ have the following form

$$
\begin{aligned}
& E_{-n}[z]=z^{-n}+\cdots+\left(a b c q^{n-1}-a-b\right) z^{n-1}, \quad n=1,2, \ldots, \\
& E_{n}[z]=z^{n}+\cdots+q^{n} z^{-n}, \quad n=1,2, \ldots
\end{aligned}
$$

Using these relation it is straightforward to prove that the non-symmetric continuous dual $q$ Hahn polynomials form a basis in $\mathcal{A}$.

Now to prove (2.10), we use the fact that the operator $Y$ acts on $\mathcal{A}$ as follows

$$
\begin{aligned}
(Y f)[z]:= & \frac{(z-c) z(1-(a+b) z+a b)}{\left(1-z^{2}\right)\left(q-z^{2}\right)}\left(f\left[q z^{-1}\right]-f[z]\right) \\
& +\frac{(1-a z)(1-b z)(1-c z)}{\left(1-z^{2}\right)\left(1-q z^{2}\right)}\left(f[q z]-f\left[z^{-1}\right]\right) .
\end{aligned}
$$

Observe that thanks to the forward shift operator relation (14.3.8) in [3], one has:

$$
q_{n}^{\dagger}(z ; a, b, c)=-\frac{q^{n} z(z-c)}{\left(q^{n}-1\right)\left(q-z^{2}\right)}\left(p_{n}(z ; a, b, c)-p_{n}\left(q^{-1} z ; a, b, c\right)\right),
$$

so that one can express $\left(Y E_{-n}\right)[z]-\frac{1}{q^{n}} E_{-n}[z]$ only in terms of $p_{n}(z ; a, b, c), p_{n}(q z ; a, b, c)$ and $p_{n}\left(q^{-1} z ; a, b, c\right)$, which can be shown to be zero by using the $q$-difference equation (14.3.7) in [3]. In a similar manner all other relations are proved.

Remark 2.5. Note that as shown in (2.12), the polynomials $E_{-n}[z]$ do not have a term of order $z^{n}$ like the non-symmetric Askey-Wilson polynomials did. This is due to the fact that the coefficient of the term $z^{n}$ in the non-symmetric Askey-Wilson polynomials tends to zero as $d \rightarrow 0$. The absence of such term makes the end of the proof of Theorem 2.2 more tricky than proof of Theorem 5.3 in [4].

Proof of Theorem 2.2. First by using the symmetry properties of the continuous dual $q$-Hahn polynomials and their properties it is easy to show that

$$
\begin{aligned}
& \left(T_{1} E_{-j}\right)[z]=-\left(1+a b-a b q^{j}\right) E_{-j}[z]-a b E_{j}[z], \\
& \left(T_{1} E_{j}\right)[z]=\left(1-q^{j}\right)\left(1-a b q^{j}\right) E_{-j}[z]-a b q^{j} E_{j}[z] .
\end{aligned}
$$

Combining this with (2.10)-(2.13), we can prove the following $\forall n>0, \forall m \in \mathbb{Z}, \forall j>0$ :

$$
\begin{aligned}
& X^{m} E_{-j}[z]=z^{m-j}+\cdots+\left(a b c q^{j-1}-a-b\right) z^{m+j-1}, \\
& X^{m} Y^{n} E_{-j}[z]=q^{-j n} z^{m-j}+\cdots+q^{-j n}\left(a b c q^{j-1}-a-b\right) z^{m+j-1}, \\
& X^{m} Y^{n} T_{1} E_{-j}[z]=-\left(1+a b-a b q^{j}\right) q^{-j n}\left(z^{m-j}+\cdots+\left(a b c q^{j-1}-a-b\right) z^{m+j-1}\right), \\
& X^{m} T_{1} E_{-j}[z]=-(1+a b) z^{m-j}+\cdots-a b z^{m+j}, \\
& X^{m} Z^{n} E_{-j}[z]=0, \\
& X^{m} Z^{n} T_{1} E_{-j}[z]=\left(\frac{-1}{a b}\right)^{n-1} q^{-n j}\left(z^{m+j}+\cdots+q^{j} z^{m-j}\right), \\
& X^{m} E_{j}[z]=z^{m+j}+\cdots+q^{j} z^{m-j}, \\
& X^{m} Y^{n} E_{j}[z]=0,
\end{aligned}
$$




$$
\begin{aligned}
& X^{m} Y^{n} T_{1} E_{j}[z]=\left(1-q^{j}\right)\left(1-a b q^{j}\right) q^{-j n}\left(z^{m-j}+\cdots+\left(a b c q^{j-1}-a-b\right) z^{m+j-1}\right), \\
& X^{m} T_{1} E_{j}[z]=\left(1-a b q^{j}-q^{j}\right) z^{m-j}-a b q^{j} z^{m+j}, \\
& X^{m} Z^{n} E_{j}[z]=\left(\frac{-1}{a b q^{j}}\right)^{n}\left(q^{j} z^{m-j}+\cdots+z^{m+j}\right), \\
& X^{m} Z^{n} T_{1} E_{j}[z]=\left(\frac{-1}{a b q^{j}}\right)^{n-1}\left(z^{m+j}+\cdots+q^{j} z^{m-j}\right) .
\end{aligned}
$$

Now assume by contradiction that a linear combination acts as zero operator in our representation, let us write such linear combination as:

$$
\begin{aligned}
& \sum_{m} a_{m} X^{m}+\sum_{m, n} b_{m, n} X^{m} Y^{n}+\sum_{m, n, i} c_{m, n} X^{m} Y^{n} T_{1}+\sum_{m, n} d_{m} X^{m} T_{1} \\
& +\sum_{m, n} e_{m, n} X^{m} Z^{n}+\sum_{m, n} f_{m, n} X^{m} Z^{n} T_{1} .
\end{aligned}
$$

Take the minimum value $M$ of $m$ such that at least one coefficient $a_{m}, b_{m, n}, c_{m, n}, d_{m}, e_{m, n}$, $f_{m, n}$ is nonzero. Acting on $E_{j}$, and collecting the terms with the minimum possible power of $z$, by (2.14) we obtain the equation:

$$
\begin{aligned}
a_{M} q^{j} & +\sum_{n} c_{M, n}\left(1-q^{j}\right)\left(1-a b q^{j}\right) q^{-j n}+d_{M}\left(1-a b q^{j}-q^{j}\right) \\
& +\sum_{n} e_{M, n}\left(\frac{-1}{a b q^{j}}\right)^{n} q^{j}+\sum_{n} f_{M, n}\left(\frac{-1}{a b q^{j}}\right)^{n-1} q^{j}=0, \quad \forall j>0 .
\end{aligned}
$$

It is easy to prove that for generic values of the parameters $a, b, c$, this is an infinite set of linearly independent equations, therefore the only possible solution is the trivial one. So we can only have coefficients of type $b_{M, n}$ not zero. Again, acting on $E_{-j}$, and collecting the terms with the minimum possible power of $z$ we obtain for every $j>0$, the equation:

$$
\sum_{n} b_{M, n} q^{-j n}=0
$$

which admit only trivial solutions.

\section{Non-symmetric Al-Salam-Chihara polynomials and basic representation of $\mathcal{H}_{\text {III }}$}

The Al-Salam-Chihara polynomials are the following:

$$
Q_{n}(z ; a, b):=\frac{(a b ; q)_{n}}{a^{n}} 3 \phi_{2}\left(\begin{array}{c}
q^{-n}, a z, a z^{-1} \\
a b, 0
\end{array} ; q, q\right),
$$

and can be obtained from the continuous dual $q$-Hahn polynomials as limits when $c \rightarrow 0$. By taking the limit $c \rightarrow 0$ of the non-symmetric continuous dual $q$-Hahn polynomials we obtain the following:

Definition 3.1. Let

$$
Q_{n}^{\dagger}(z ; a, b):=q^{\frac{n-1}{2}} z Q_{n-1}\left(q^{-\frac{1}{2}} z ; q^{\frac{1}{2}} a, q^{\frac{1}{2}} b\right),
$$

the non-symmetric Al-Salam-Chihara polynomials are defined as follows:

$$
\begin{aligned}
& E_{-n}[z]:=Q_{n}(z ; a, b)-Q_{n}^{\dagger}(z ; a, b), \quad n=1,2, \ldots, \\
& E_{n}[z]:=q^{n} Q_{n}(z ; a, b)+\left(1-q^{n}\right) Q_{n}^{\dagger}(z ; a, b), \quad n=1,2, \ldots, \\
& E_{0}[z]:=1 .
\end{aligned}
$$


Theorem 3.2. For $q, a, b \neq 0, q^{m} \neq 1(m=1,2, \ldots)$, the algebra $\mathcal{H}_{\mathrm{III}}$ has a faithful representation on the space $\mathcal{A}$ of Laurent polynomials $f[z]$ as follows:

$$
\begin{aligned}
& \left(T_{0} f\right)[z]:=-\frac{z}{q-z^{2}}\left(f[z]-f\left[q z^{-1}\right]\right), \\
& \left(T_{1} f\right)[z]:=\frac{(a+b) z-(1+a b)}{1-z^{2}} f[z]+\frac{(1-a z)(1-b z)}{1-z^{2}} f\left[z^{-1}\right], \\
& (X f)[z]:=z f[z] .
\end{aligned}
$$

To prove that the operators defined by (3.1)-(3.3) satisfy the relations (1.5)-(1.8) is a straightforward computation. To prove faithfulness, we again need to provide an equivalent representation for the algebra $\mathcal{H}_{\text {III }}$. This is where we need to be careful as the relation between the non-symmetric Al-Salam-Chihara polynomials and the algebra $\mathcal{H}_{\mathrm{III}}$ is not as straightforward as before because the algebra $\mathcal{H}_{\mathrm{III}}$ was obtained as limit of $\mathcal{H}_{\mathrm{V}}$ as $c \rightarrow \infty$ rather than $c \rightarrow 0$. However, changing the definition of $Z$ and $Y$ we can still prove the following:

Lemma 3.3. Let

$$
Z:=-X T_{0} T_{1}^{-1}+T_{1}^{-1} \quad \text { and } \quad Y:=-T_{1} X T_{0},
$$

then the algebra $\mathcal{H}_{\mathrm{III}}$ can equivalently be described as the algebra generated by $T_{1}, X^{ \pm 1}, Y, Z$, satisfying the following relations respectively:

$$
\begin{aligned}
& Z Y=Y Z=0, \\
& X T_{1}=-a b T_{1}^{-1} X^{-1}-a-b, \\
& T_{1}^{-1} Y=Z T_{1}-1, \\
& \left(T_{1}+a b\right)\left(T_{1}+1\right)=0, \\
& a b Y X=-q T_{1}^{2} X Y-q(a+b) T_{1} Y-a b T_{1} X .
\end{aligned}
$$

The algebra $\mathcal{H}_{\mathrm{III}}$ is spanned by elements $X^{m} Y^{n} T_{1}^{i}$ and $X^{m} Z^{n} T_{1}^{i}$, where $m \in \mathbb{Z}, n \in \mathbb{N}$ and $i=1,2$.

Proof. The relations (3.5)-(3.9) follow from (1.5)-(1.8). Vice-versa, defining $T_{0}:=-X^{-1} T_{1}^{-1} Y$ we see that relations (3.5)-(3.9) imply (1.5)-(1.8).

To prove that $\mathcal{H}_{\text {III }}$ is spanned by elements $X^{m} Y^{n} T_{1}^{i}$ and $X^{m} Z^{n} T_{1}^{i}$, where $m \in \mathbb{Z}, n \in \mathbb{N}$ and $i=1,2$, we use the relations (3.5)-(3.9) and the further equivalent relations

$$
\begin{aligned}
& Y X^{-1}=q^{-1} X^{-1} Y+q^{-1}(1+a b) X^{-1} Z T_{1}-q^{-1}(a+b) Z T_{1}+q^{-1} X^{-1} T_{1}, \\
& Z X=q^{-1} X Z-q \frac{1+a b}{a b} X^{-1} Z T_{1}+\frac{1+a b}{a b} Z T_{1}-\frac{1}{a b} X^{-1} T_{1}+\frac{(1+a b)(q-1)}{a b} X^{-1}
\end{aligned}
$$

to order any word in the algebra as wanted.

Lemma 3.4. The non-symmetric Al-Salam-Chihara polynomials form a basis in the space $\mathcal{A}$ of Laurent polynomials and are eigenfunctions of the operators $Y$ and $Z$ :

$$
\begin{aligned}
& \left(Y E_{-n}\right)[z]=\frac{1}{q^{n}} E_{-n}[z], \quad n=1,2, \ldots, \\
& \left(Y E_{n}\right)[z]=0, \quad n=0,1,2, \ldots \\
& \left(Z E_{-n}\right)[z]=0, \quad n=0,1,2, \ldots, \\
& \left(Z E_{n}\right)[z]=-\frac{1}{a b q^{n}} E_{n}[z], \quad n=1,2, \ldots
\end{aligned}
$$


Proof. Consider the following isomorphism:

$$
\eta\left(T_{0}, T_{1}, X\right)=\left(-X T_{0}, T_{1}, X\right)=\left(\tilde{T}_{0}, \tilde{T}_{1}, \tilde{X}\right)
$$

which maps the algebra $\mathcal{H}_{\text {III }}$ to the isomorphic algebra $\tilde{\mathcal{H}}_{\text {III }}$ defined by the generators $\tilde{T}_{0}, \tilde{T}_{1}, \tilde{X}$ and relations

$$
\begin{aligned}
& \left(\tilde{T}_{1}+a b\right)\left(\tilde{T}_{1}+1\right)=0, \quad \tilde{T}_{0}^{2}+\tilde{T}_{0}=0, \\
& \left(\tilde{T}_{1} \tilde{X}+a\right)\left(\tilde{T}_{1} \tilde{X}+b\right)=0, \quad q \tilde{T}_{0} \tilde{X}^{-1}=\tilde{X}\left(\tilde{T}_{0}+1\right) .
\end{aligned}
$$

Note that the algebra $\tilde{\mathcal{H}}_{\mathrm{III}}$ is obtained by taking the limit of $c \rightarrow 0$ of the algebra $\mathcal{H}_{\mathrm{V}}$, so that the proof of this lemma is based on the fact that the action of the new $Y$ and $Z$ defined by (3.4) is obtained by taking the limit of $c \rightarrow 0$ of the corresponding action of the old $Y$ and $Z$ defined in Section 2.

Proof of Theorem 3.2. Similarly to the proof of Lemma 3.4, we can use the isomorphism $\eta$ to prove this theorem by taking the limit $c \rightarrow 0$ of the proof of Theorem 2.2 - note that this limit none of the coefficients in the relations (2.14) becomes zero, thus making this limit rather straight-forward.

\section{Non-symmetric continuous (big) $q$-Hermite polynomials and basic representations of $\left(\mathcal{H}_{\text {III }}^{D_{7}}\right) \mathcal{H}_{\text {III }}^{D_{8}}$}

In this section we give all definitions and proof for the symmetric continuous big $q$-Hermite polynomials and the algebra $\mathcal{H}_{\mathrm{III}}^{D_{7}}$. By taking the simple limit $a \rightarrow 0$, all proofs remain valid for the $\mathcal{H}_{\mathrm{III}}^{D_{8}}$ algebra and the continuous $q$-Hermite polynomials.

The continuous big $q$-Hermite polynomials are the following:

$$
H_{n}(z ; a):=z_{2}^{n} \phi_{0}\left(\begin{array}{c}
q^{-n}, a z \\
-
\end{array} q, q^{n} z^{-2}\right),
$$

and can be obtained from the Al-Salam-Chihara polynomials as limits when $b \rightarrow 0$. By taking the limit $b \rightarrow 0$ of the non-symmetric continuous dual Al-Salam-Chihara we obtain the following:

Definition 4.1. Let

$$
Q_{n}^{\dagger}(z ; a):=q^{\frac{n-1}{2}} z H_{n-1}\left(q^{-\frac{1}{2}} z ; q^{\frac{1}{2}} a\right)
$$

the non-symmetric continuous big $q$-Hermite polynomials are defined as follows:

$$
\begin{aligned}
& E_{-n}[z]:=H_{n}(z ; a)-Q_{n}^{\dagger}(z ; a), \quad n=1,2, \ldots, \\
& E_{n}[z]:=q^{n} H_{n}(z ; a)+\left(1-q^{n}\right) Q_{n}^{\dagger}(z ; a), \quad n=1,2, \ldots, \\
& E_{0}[z]:=1 .
\end{aligned}
$$

Similarly, the non-symmetric continuous $q$-Hermite polynomials are defined by taking the limit of the non-symmetric continuous big $q$-Hermite polynomials as $a \rightarrow 0$.

Theorem 4.2. For $q, a \neq 0, q^{m} \neq 1(m=1,2, \ldots)$, the algebra $\mathcal{H}_{\mathrm{III}}^{D_{7}}$ has a faithful representation on the space $\mathcal{A}$ of Laurent polynomials $f[z]$ as follows:

$$
\left(T_{0} f\right)[z]:=-\frac{z}{q-z^{2}}\left(f[z]-f\left[q z^{-1}\right]\right)
$$




$$
\begin{aligned}
& \left(T_{1} f\right)[z]:=\frac{a z-1}{1-z^{2}}\left(f[z]-f\left[z^{-1}\right]\right), \\
& (X f)[z]:=z f[z] .
\end{aligned}
$$

By taking the above representation for $a=0$ ( still assuming $q \neq 0, q^{m} \neq 1$ for $m=1,2, \ldots$ ), we obtain a faithful representation of the algebra $\mathcal{H}_{\mathrm{III}}^{D_{8}}$.

To prove that the operators defined by (4.1)-(4.3) satisfy the relations (1.9)-(1.12) is a straightforward computation. To prove faithfulness, we can't use an equivalent representation à la Bernstein-Zelevinsky as there isn't one. We proceed by proving the following two lemmata:

Lemma 4.3. The algebras $\mathcal{H}_{\mathrm{III}}^{D_{7}}$ and $\mathcal{H}_{\mathrm{III}}^{D_{8}}$ are spanned by the elements

$$
X^{k}\left(T_{0} T_{1}\right)^{l}, \quad X^{k}\left(T_{0} T_{1}\right)^{l} T_{0}, \quad X^{k}\left(T_{1} T_{0}\right)^{l}, \quad X^{k}\left(T_{1} T_{0}\right)^{l} T_{1} \quad \text { for } \quad k \in \mathbb{Z}, l \in \mathbb{N} .
$$

Proof. We give the proof for the algebra $\mathcal{H}_{\mathrm{III}}^{D_{7}}$ only, as the limit $a \rightarrow 0$ in this proof is a straightforward substitution of $a$ by 0 .

Let us consider all possible words in the algebra $\mathcal{H}_{\mathrm{III}}^{D_{7}}$ and order them by using relations (1.11) and (1.12) in such a way that all powers of $X$ are on the left. Thanks to (1.9) and (1.10) the generators $T_{0}$ and $T_{1}$ may only appear with powers 1 or 0 . We then are the following possible words:

$$
X^{k}\left(T_{0} T_{1}\right)^{l}, \quad X^{k}\left(T_{0} T_{1}\right)^{l} T_{0}, \quad X^{k}\left(T_{1} T_{0}\right)^{l}, \quad X^{k}\left(T_{1} T_{0}\right)^{l} T_{1} \quad \text { for } \quad k \in \mathbb{Z}, l \in \mathbb{N},
$$

as we wanted to prove.

Lemma 4.4. The non-symmetric big q-Hermite polynomials form a basis in the space $\mathcal{A}$ of Laurent polynomials and the operators $T_{0}$ and $T_{1}$ act on them as follows:

$$
\begin{aligned}
& \left(T_{0} E_{j}\right)[z]=0, \\
& \left(T_{0} E_{-j}\right)[z]=-\frac{1}{q^{j}} E_{j-1}[z], \\
& \left(T_{1} E_{j}\right)[z]=\left(1-q^{j}\right) E_{-j}[z], \\
& \left(T_{1} E_{-j}\right)[z]=-E_{-j}[z] .
\end{aligned}
$$

Proof. By using the definition of the $q$-hypergeometric series ${ }_{2} \phi_{0}$ it is easy to prove that the terms with the highest powers in $z$ and $\frac{1}{z}$ in $E_{-n}$ and $E_{n}$ have the following form

$$
\begin{aligned}
& E_{-n}[z]=z^{-n}+\cdots-a z^{n-1}, \quad n=1,2, \ldots, \\
& E_{n}[z]=z^{n}+\cdots+q^{n} z^{-n}, \quad n=1,2, \ldots
\end{aligned}
$$

Using these relations it is straightforward to prove that the non-symmetric big $q$-Hermite polynomials form a basis in $\mathcal{A}$.

To prove (4.4)-(4.7) we use the recurrence relation of the big $q$-Hermite polynomials combined with the forward shift relation.

Proof of Theorem 4.2. To prove faithfulness we first look at how the operators $X^{k}\left(T_{0} T_{1}\right)^{l}$, $X^{k}\left(T_{0} T_{1}\right)^{l} T_{0}, X^{k}\left(T_{1} T_{0}\right)^{l}$ and $X^{k}\left(T_{1} T_{0}\right)^{l} T_{1}$ act on the non-symmetric big $q$-Hermite polynomials for every $k \in \mathbb{Z}, l \in \mathbb{N}$. To this aim, using (4.4)-(4.6) one can prove the following relations:

$$
\left(X^{k}\left(T_{0} T_{1}\right)^{l} E_{j}\right)[z]=-\frac{1-q^{j}}{q^{j}}\left(X^{k}\left(T_{0} T_{1}\right)^{l-1} E_{j-1}\right)[z], \quad \forall j>0,
$$




$$
\begin{aligned}
& \left(X^{k}\left(T_{0} T_{1}\right)^{l} E_{-j}\right)[z]=\frac{1}{q^{j}}\left(X^{k}\left(T_{0} T_{1}\right)^{l-1} E_{j-1}\right)[z], \quad \forall j>0, \\
& \left(X^{k}\left(T_{0} T_{1}\right)^{l} T_{0} E_{-j}\right)[z]=\frac{1}{q^{j}} \frac{1-q^{j-1}}{q^{j-1}}\left(X^{k}\left(T_{0} T_{1}\right)^{l-1} E_{j-2}\right)[z], \quad \forall j>1, \\
& \left(X^{k}\left(T_{1} T_{0}\right)^{l} E_{-j}\right)[z]=-\frac{1-q^{j-1}}{q^{j}}\left(X^{k}\left(T_{1} T_{0}\right)^{l-1} E_{-j+1}\right)[z], \quad \forall j>0, \\
& \left(X^{k}\left(T_{1} T_{0}\right)^{l} T_{1} E_{j}\right)[z]=-\frac{1-q^{j}}{q^{j}}\left(1-q^{j-1}\right)\left(X^{k}\left(T_{1} T_{0}\right)^{l-1} E_{-j+1}\right)[z], \quad \forall j>0, \\
& \left(X^{k}\left(T_{1} T_{0}\right)^{l} T_{1} E_{-j}\right)[z]=\frac{1-q^{j-1}}{q^{j}}\left(X^{k}\left(T_{1} T_{0}\right)^{l-1} E_{-j+1}\right)[z], \quad \forall j>0 .
\end{aligned}
$$

By iteration it is straight-forward to obtain:

$$
\begin{aligned}
& \left(X^{k}\left(T_{0} T_{1}\right)^{l} E_{j}\right)[z]=(-1)^{l} \frac{\left(q^{j-l+1} ; q\right)_{l}}{q^{\frac{l(1+2 j-l)}{2}}}\left(X^{k} E_{j-l}\right)[z], \quad \forall j \geq l, \\
& \left(X^{k}\left(T_{0} T_{1}\right)^{l} E_{-j}\right)[z]=(-1)^{l-1} \frac{\left(q^{j-l+1} ; q\right)}{q^{\frac{l(1+2 j-l)}{2}}}\left(X^{k} E_{j-l}\right)[z], \quad \forall j \geq l, \\
& \left(X^{k}\left(T_{0} T_{1}\right)^{l} T_{0} E_{-j}\right)[z]=(-1)^{l-1} \frac{\left(q^{j-l} ; q\right)_{l}}{q^{\frac{(l+1)(2 j-l)}{2}}}\left(X^{k} E_{j-l-1}\right)[z], \quad \forall j>l, \\
& \left(X^{k}\left(T_{1} T_{0}\right)^{l} E_{-j}\right)[z]=(-1)^{l} \frac{\left(q^{j-l} ; q\right)_{l}}{q^{\frac{l(1+2 j-l)}{2}}}\left(X^{k} E_{-j+l}\right)[z], \quad \forall j \geq l, \\
& \left(X^{k}\left(T_{1} T_{0}\right)^{l} T_{1} E_{j}\right)[z]=(-1)^{l} \frac{\left(q^{j-l} ; q\right)_{l+1}}{q^{\frac{l(1+2 j-l)}{2}}}\left(X^{k} E_{-j+l}\right)[z], \quad \forall j \geq l, \\
& \left(X^{k}\left(T_{1} T_{0}\right)^{l} T_{1} E_{-j}\right)[z]=(-1)^{l-1} \frac{\left(q^{j-l} ; q\right)}{q^{\frac{l(1+2 j-l)}{2}}}\left(X^{k} E_{-j+l}\right)[z], \quad \forall j \geq l .
\end{aligned}
$$

Combining these with (4.8) and (4.9), we obtain the following estimates $\forall j>l$ :

$$
\begin{aligned}
& \left(X^{k}\left(T_{0} T_{1}\right)^{l} E_{j}\right)[z]=(-1)^{l} \frac{\left(q^{j-l+1} ; q\right)_{l}}{q^{\frac{l(1+2 j-l)}{2}}}\left(z^{k+j-l}+\cdots+q^{j-l} z^{k-j+l}\right), \\
& \left(X^{k}\left(T_{0} T_{1}\right)^{l} E_{-j}\right)[z]=(-1)^{l-1} \frac{\left(q^{j-l+1} ; q\right)_{l-1}}{q^{\frac{l(1+2 j-l)}{2}}}\left(z^{k+j-l}+\cdots+q^{j-l} z^{k-j+l}\right), \\
& \left(X^{k}\left(T_{0} T_{1}\right)^{l} T_{0} E_{-j}\right)[z]=(-1)^{l-1} \frac{\left(q^{j-l} ; q\right)_{l}}{q^{\frac{(l+1)(2 j-l)}{2}}}\left(z^{k+j-l-1}+\cdots+q^{j-l} z^{k-j+l+1}\right), \\
& \left(X^{k}\left(T_{1} T_{0}\right)^{l} E_{-j}\right)[z]=(-1)^{l} \frac{\left(q^{j-l} ; q\right)_{l}}{q^{\frac{l(1+2 j-l)}{2}}\left(z^{k-j+l}+\cdots-a z^{k+j-l-1}\right),} \\
& \left(X^{k}\left(T_{1} T_{0}\right)^{l} T_{1} E_{j}\right)[z]=(-1)^{l} \frac{\left(q^{j-l} ; q\right)_{l+1}}{q^{\frac{l(1+2 j-l)}{2}}}\left(z^{k-j+l}+\cdots-a z^{k+j-l-1}\right), \\
& \left(X^{k}\left(T_{1} T_{0}\right)^{l} T_{1} E_{-j}\right)[z]=(-1)^{l-1} \frac{\left(q^{j-l} ; q\right)_{l}}{q^{\frac{l(1+2 j-l)}{2}}}\left(z^{k-j+l}+\cdots-a z^{k+j-l-1}\right) .
\end{aligned}
$$

Now assume by contradiction that a linear combination acts as zero operator in our representation, let us write such linear combination as:

$$
\sum_{k, l} a_{k, l} X^{k}\left(T_{0} T_{1}\right)^{l}+\sum_{k, l} b_{k, l} X^{k}\left(T_{0} T_{1}\right)^{l} T_{0}+\sum_{k, l} c_{k, l} X^{k}\left(T_{1} T_{0}\right)^{l}+\sum_{k, l} d_{k, l} X^{k}\left(T_{1} T_{0}\right)^{l} T_{1} .
$$


Take the minimum value $k_{0}$ of $k$ such that at least one coefficient $a_{k_{0}, l}, b_{k_{0}, l}, c_{k_{0}, l}, d_{k_{0}, l}$ is nonzero. Acting on $E_{j}[z]$, for all $j>l$, and collecting the terms with the minimum possible power of $z$, which is $z^{k_{0}-j+l}$, we obtain the equation:

$$
\sum_{l} a_{k_{0}, l}(-1)^{l} \frac{\left(q^{j-l+1} ; q\right)_{l}}{q^{\frac{l(1+2 j-l)}{2}}} q^{j-l}+\sum_{l} d_{k_{0}, l}(-1)^{l} \frac{\left(q^{j-l} ; q\right)_{l+1}}{q^{\frac{l(1+2 j-l)}{2}}}=0, \quad \forall j>l .
$$

It is easy to prove that for generic values of $a$, this is an infinite set of linearly independent equations, therefore the only possible solution is the trivial one, i.e. $a_{k_{0}, l}=0, d_{k_{0}, l}=0$ for all values of $l$.

By acting on $E_{j}[z]$, we can prove in a similar way that $b_{k_{0}, l}=0, c_{k_{0}, l}=0$ for all values of $l$, therefore obtaining a contradiction.

To prove the same for the algebra $\mathcal{H}_{\text {III }}^{D_{8}}$ we observe that the defining relations $(1.13)-(1.16)$ are a specialisation of the defining relations (1.9)-(1.12) of the algebra $\mathcal{H}_{\mathrm{III}}^{D_{7}}$ for $a=0$. All results hold true when $a \rightarrow 0$. Indeed even if the polynomials $E_{n}$ loose the terms of order $z^{n-1}$, these don't enter in the above reasoning. This concludes the proof of our theorem.

\section{Acknowledgements}

The author is specially grateful to T. Koornwinder and J. Stokman for interesting discussions on this subject and the referees for pointing out references, typos and giving suggestions on how to improve the presentation of the main results.

\section{References}

[1] Askey R., Wilson J., Some basic hypergeometric orthogonal polynomials that generalize Jacobi polynomials, Mem. Amer. Math. Soc. 54 (1985), iv+55 pages.

[2] Cherednik I., Double affine Hecke algebras, Knizhnik-Zamolodchikov equations, and Macdonald's operators, Int. Math. Res. Not. 1992 (1992), no. 9, 171-180.

[3] Koekoek R., Lesky P.A., Swarttouw R.F., Hypergeometric orthogonal polynomials and their $q$-analogues, Springer Monographs in Mathematics, Springer-Verlag, Berlin, 2010.

[4] Koornwinder T.H., The relationship between Zhedanov's algebra AW(3) and the double affine Hecke algebra in the rank one case, SIGMA 3 (2007), 063, 15 pages, math.QA/0612730.

[5] Mazzocco M., Confluences of the Painlevé equations, Cherednik algebras and $q$-Askey scheme, arXiv:1307.6140.

[6] Noumi M., Stokman J.V., Askey-Wilson polynomials: an affine Hecke algebra approach, in Laredo Lectures on Orthogonal Polynomials and Special Functions, Adv. Theory Spec. Funct. Orthogonal Polynomials, Nova Sci. Publ., Hauppauge, NY, 2004, 111-144, math.QA/0001033.

[7] Sahi S., Nonsymmetric Koornwinder polynomials and duality, Ann. of Math. 150 (1999), 267-282, q-alg/9710032. 